\title{
Non-coding yet non-trivial: a review on the computational genomics of lincRNAs
}

\author{
Travers Ching ${ }^{1,2}$, Jayson Masaki ${ }^{3}$, Jason Weirather ${ }^{4}$ and Lana X. Garmire ${ }^{1,2^{*}}$
}

\author{
* Correspondence: \\ Igarmire@cc.hawaii.edu \\ ${ }^{1}$ Molecular Biosciences and \\ Bioengineering Graduate Program, \\ University of Hawaii at Manoa, \\ Honolulu, HI 96822, USA \\ Epidemiology Program, University \\ of Hawaii Cancer Center, Honolulu, \\ HI 96813, USA \\ Full list of author information is \\ available at the end of the article
}

\begin{abstract}
Long intergenic non-coding RNAs (lincRNAs) represent one of the most mysterious RNA species encoded by the human genome. Thanks to next generation sequencing (NGS) technology and its applications, we have recently witnessed a surge in non-coding RNA research, including lincRNA research. Here, we summarize the recent advancement in genomics studies of lincRNAs. We review the emerging characteristics of lincRNAs, the experimental and computational approaches to identify lincRNAs, their known mechanisms of regulation, the computational methods and resources for lincRNA functional predictions, and discuss the challenges to understanding lincRNA comprehensively.
\end{abstract}

\section{Introduction}

The mainstream focus of biomedical research has been in elucidating the functions and interactions among proteins within the cell. In line with the central dogma of molecular biology, RNAs were once perceived as the intermediary for protein production and the archaic precursor molecule of DNA. However, RNAs are transcribed from more than $85 \%$ of genomic regions [1], whereas proteins are only encoded in less than $3 \%$ of human genome sequences [2]. This leaves a mysterious knowledge gap in either the efficiency of cellular transcription to translation or a foundational misunderstanding in gene expression regulation and RNA function. It was thought that RNAs had limited but essential and evolutionarily common roles of basic cell machinery such as tRNA, rRNA, and mRNA. The few examples of functional RNAs or RNAs with enzymatic-like activity, were considered as evolutionary remnants [3]. For a long period of time, non-coding RNA (ncRNA) transcripts were believed to be by-products derived from mRNA degradation or nonspecific polymerase activity, and therefore termed "transcriptional noise" [4].

It is now becoming evident that ncRNAs are responsible for many aspects of gene regulation. Some small non-coding RNAs, such as microRNAs, siRNAs, snRNAs, snoRNAs, exRNAs and piRNAs, have been well categorized over the past decade. However, long noncoding RNAs (lncRNAs) remained relatively unexplored due to the challenges of computational prediction under poor sequence conservation and low homology within the set of lncRNAs. Some of these challenges have been addressed by the revolutionary inventions of next generation sequencing (NGS) and its applications, such as RNA-Seq, which captures whole transcriptome data, including lncRNAs. Among the human lncRNAs, tens of thousands of long intergenetic noncoding RNAs (lincRNAs) 
have been discovered in the genomic regions outside of the well-studied coding genomic regions, and they show many intriguing properties, such as associations with various human diseases, tissue-specific expression, and expression changes during development. Consequently, attributing organism complexity to the hidden regulation of lincRNAs is a fascinating new area of research. Here, we review the emerging characteristics of lincRNAs; the experimental and computational approaches to identifying lincRNAs and their mechanisms of regulation; the challenges in computational predictions; and the resources still required to advance our understanding of lincRNA-related genomic regulation.

\section{Review}

\section{Emerging characteristics of lincRNAs}

LincRNAs are a putatively heterogeneous group, conventionally defined as ncRNA transcripts of more than $200 \mathrm{bp}$ located in regions with no overlap to any known proteincoding genes. According to Lncipedia, a comprehensive lncRNA database, high-throughput studies of transcriptome data have catalogued over 111,000 lncRNA transcripts, with roughly $50 \%$ coming from intergenic regions [5]. The majority of lincRNAs are thought to be transcribed from RNA polymerase II, and are therefore usually modified by post-transcriptional 5' capping and 3' polyadenylation [6]. Surprisingly, lincRNAs show ribosome occupancy similar to the 5'UTRs of protein coding genes [7]. What differentiates lincRNAs from protein coding genes seems to be the lack of release upon encountering a stop codon in the lincRNA sequences [7]. Therefore, polyadenlyation and 5' capping are not necessarily markers of protein coding functionality. However, lincRNAs show a markedly higher degree of tissue-specific [8] and disease specific expression [9], suggesting some biological function.

LincRNA expression is generally much lower than protein coding genes, with a few exceptions such as the XIST lincRNA [10]. For some lincRNAs, even just a few or a single transcript exist in a cell, determined by RNA-Seq data [10]. However, rather than being spurious by-products of non-specific RNA transcription, the expression levels of lincRNAs in any given cell are precisely coordinated throughout the tissue, and dynamic through the course of development [11]. Researchers have detected differential expression of lincRNA in a range of tissues, diseases, and specific cellular responses. Efforts have been made to take advantage of these properties of lincRNAs for translational and clinical applications, such as disease biomarkers [12].

Another unique feature of lincRNAs is the low sequence conservation. LincRNAs exhibit $22-25 \%$ of conserved bases under purifying selection, compared to $77 \%$ in protein coding sequences. However, they are considerably more conserved than introns, which have $7 \%$ conservation [13]. Under the assumption that sequence conservation reflects biological significance, the high genomic sequence variability in lincRNAs was the initial basis to call them "junk RNAs". Unlike proteins, where evolutionary conservation correlates highly with functional importance, lincRNAs seem to be under different selective pressures. Many lincRNA are predicted to have secondary structure and may therefore act in a sequence independent manner [14]. Consequently, there may be a greater functional importance on molecular 3D conformation over the primary sequence. This is supported by a recent global study of genetic variants in human lincRNAs in association with diseases, where single nucleotide polymorphisms (SNPs) in evolutionarily conserved regions of lincRNAs had significant effects on predicted secondary structure [15]. 


\section{Genome-wide detection of lincRNAs}

Chromatin immunoprecipitation sequencing (ChIP-Seq) is an NGS method that has allowed the discovery of global genomic binding sites of DNA-interacting proteins, such as transcription factors and histones. Using ChIP-Seq signatures of histone 3 lysine 4 tri-methylation (H3K4me3) and histone 3 lysine 36 tri-methylation (HK36me3), or so called "K4-K36" clusters, Guttman et al. detected approximately 1700 transcriptional units $>5 \mathrm{~kb}$ among four mouse cell lines, which were confirmed by tiling microarrays, PCR and northern blots [16]. This type of chromatin signature was later applied to human cell lines to identify lincRNAs and was shown that along with HOTAIR, $20 \%$ of lincRNAs were associated with the Polycomb repressive complexes 2 (PRC2) [4]. ChIP-Seq has also been applied to the detection of RNA pol II occupancy to identify lincRNAs in mouse macrophages upon endotoxin stimulation [17]. The authors found that $70 \%$ of extragenic polymerase II peaks were associated with genomic regions with a canonical chromatin signature of enhancers.

Clearly, decisions made during the library preparation phase of an RNA-seq experiment will affect lincRNA measurements. Since many but not all lincRNA transcripts are poly-adenylated [18], the decision to select poly-adenylated RNAs or to use ribodepletion methods should be made with care. Yang et al. [19] state that approximately $20 \%$ of transcripts are non-poly adenylated, suggesting that ribo-depletion methods are necessary to gain a more comprehensive picture of the transcriptome. In addition, Yang et al. find that some transcripts, such as the Malat1 lincRNA are bimorphic, meaning they exist in poly-A(+) and poly-A(-) configurations. Thus, ribo-depletion and poly-A selection methods could provide complementary information on the relative proportions of poly-adenylation of transcripts. Moreover, the adoption of strand-specific sequencing protocols provides a means of making more detailed annotations of lncRNAs, especially the antisense lncRNAs [20]. Nevertheless, even without strand information, RNA-seq has proven useful for the identification of lincRNAs. For example, Cabili et al. analysed lincRNAs in 24 tissues and mapped out nearly 9000 lincRNAs coupled to expression profile information [8].

Not all NGS methods are ideal for identifying the precise boundaries of lincRNAs. ChIP-Seq using antibodies against RNA polymerases can only provide a rough estimation of transcription location but not the precise boundaries of transcripts [17]. RNA-Seq may also have trouble to detect isoforms and their exact start and end sites, as the cDNA is randomly fragmented, and accumulated from all isoforms within a given genomic loci [21]. Moreover, if RNA-Seq is conducted by a poly-A enriched approach, the internal bias against $5^{\prime}$ ends make it difficult to map out the exact start sites of a transcript. However, some other NGS methods have been adopted to overcome this problem. For example, cap analysis gene expression (CAGE) tag sequencing has been used to aid the identification of transcription start sites in human cells [18], and 3'-end sequencing (3SEQ) has also been used in a zebrafish model to aid the determination of the 3' bounds of lincRNA transcripts [22]. Additionally, tiling arrays that enable direct observation of lincRNAs transcript exons have been used to detect gene boundaries and alternative splicing. For example, Tahira et al. sampled intergenic and intronic ESTs from over one million ESTs from The Cancer Genome Project to develop a custom microarray, and subsequently identified lincRNAs differentially expressed between primary and metastatic pancreatic cancers [23]. 


\section{Computational methods to predict lincRNAs}

Most computational studies of lincRNAs rely on RNA-Seq results initially, with quality-control filtering steps to remove reads arising from spurious background noise [24]. Additional steps should be taken involving the removal of protein coding genes and small non-coding RNAs such as microRNAs. Methods to do such removals include ORF detection [1, 9, 16, 25], BLAST to identify homologs of protein coding genes [25], domain based searches such as Pfam $[9,25]$, and predictions of coding potential based on nucleotide substitution frequencies given sequences from multiple species. The Coding Potential Calculator (CPC) [26] and iSeeRNA [27] programs are popular choices in determining coding potential. However, the extent to which some lincRNAs may be hosts of smaller RNA species such as microRNAs requires further study [28]. Another selection criterion is the number of exons in a transcript. Most of the exons (about $80 \%$ in human) are less than 200 bp [29], the minimum length requirement of lincRNA by definition. Transcripts with only one exon are less likely to be lincRNAs. Additionally, the number of exons can be used as an indicator of transcript quality. Multi-exonic transcripts are less likely to result from spurious transcription and genomic noise. The presence of introns is also indicative of robust and consistent transcription boundaries. Introns have less frequent terminal repeats and transposable elements in comparison to intergenic regions, suggesting that lincRNAs have additional conservation in splicing [30]. Finally, the axiomatic length-based filter, 200 bp, eliminates any non-coding sequences that fall into the current small RNA categories [31]. The filtering steps described above are often implemented through a pipeline with a series of cut-offs or a decision tree to interrogate multiple features involved in classifying lincRNAs [24].

In recent years, machine learning based classification approaches have been used to detect lincRNAs [17, 27, 32-34]. For example, iSeeRNA interrogated coding potential based on a variety of factors mentioned above, in addition to nucleotide composition. It was trained to differentiate protein coding genes and lncRNAs with an area under the curve (AUC) of 0.99 [27]. LncRNA-MFDL is another tool that uses a deep learning method and the fusion of multiple features to classify lincRNAs with an accuracy of $97.1 \%[34]$.

\section{lincRNA databases}

LincRNAs identified from exploratory studies are a valuable resource for accumulating information about these relatively unknown transcripts. Information such as location, splice junction, and tissue specificity are important features. There are quite a few specialized databases that provide comprehensive annotations for lincRNAs or lncRNAs. These include The Broad Institute's Human Body Map project [4], NONCODE [35] and Lncipedia [5]. Other large gene annotation sets such as GENCODE [36, 37], UCSC's known genes [38] or Rfam [39] RNA family databases are not specific to non-coding RNAs, but nevertheless contain large sets of annotations and information on lincRNAs.

The UCSC ENCODE project provides a feature-rich resource to describe the transcriptional landscape in a variety of tissues from the GENCODE database [40]. The Ensembl Geneome Browser is another resource that identifies and annotates transcripts 
within their large database using transcriptional evidence as well as chromatin markups [36]. The Ensembl project uses the GENCODE database, and contributes multiple sources to GENCODE through an automated annotation pipeline in combination with the large Havana annotation by the Sanger Institute [36]. While GENCODE is one of the most comprehensive databases for mammalian species, it does not include lincRNAs found by RNA-Seq ab initio alignment methods, such as those in the Human Body Map. Neither is it as comprehensive as specialized databases.

More specialized lncRNA databases, such as NONCODE and Lncipedia, enumerate a much larger number of lncRNAs (Table 1). These databases have been created to facilitate functional analyses by integrating multiple data sources such as expression, chromatin markups, microRNA binding sites and mutational data with known lncRNAs. Not surprisingly, the overlap of those data sets can differ greatly, largely due to the selection criteria of particular lncRNAs or the tissue origins where lincRNAs were initially detected.

Table 1 Summary of IncRNA/lincRNA databases

\begin{tabular}{|c|c|c|}
\hline Project Name & Species & Purpose \\
\hline Human Body Map & Human & A reference set of lincRNAs \\
\hline ChIPBase & $\begin{array}{l}\text { Various (incl. Human } \\
\text { and Mouse) }\end{array}$ & $\begin{array}{l}\text { A resource for IncRNA transcriptional regulation and expression } \\
\text { profiles of ncRNA (IncRNA, microRNAs, etc.) }\end{array}$ \\
\hline NONCODE & $\begin{array}{l}\text { Various (incl. Human } \\
\text { and Mouse) }\end{array}$ & $\begin{array}{l}\text { A large IncRNA database integrating various databases and } \\
\text { references }\end{array}$ \\
\hline IncRNAdb & $\begin{array}{l}\text { Various (incl. Human } \\
\text { and Mouse) }\end{array}$ & $\begin{array}{l}\text { A database of IncRNAs having biological function or regulatory } \\
\text { roles }\end{array}$ \\
\hline $\begin{array}{l}\text { ncRNA expression } \\
\text { database (NRED) }\end{array}$ & Human and Mouse & Expression database for human and mouse IncRNAs \\
\hline LNCipedia & $\begin{array}{l}\text { Various (incl. Human } \\
\text { and Mouse) }\end{array}$ & A large database of IncRNA transcripts and annotation \\
\hline LncRNADisease & Human & A database of IncRNAs associated with human diseases \\
\hline DIANA-LncBase & Human and Mouse & $\begin{array}{l}\text { A database of experimentally verified and predicted microRNA } \\
\text { targets on IncRNAs }\end{array}$ \\
\hline IncRNA2Target & Human and Mouse & $\begin{array}{l}\text { A collection of IncRNA knockout experiments and } \\
\text { downstream regulation }\end{array}$ \\
\hline starBase 2.0 & $\begin{array}{l}\text { Human, Mouse and } \\
\text { C. elegans }\end{array}$ & $\begin{array}{l}\text { A collection of IncRNA and predicted microRNA targets; IncRNA } \\
\text { expression profiles from TCGA data }\end{array}$ \\
\hline IncRNAMap & Human & $\begin{array}{l}\text { A resource for exploring IncRNA expression profiles and } \\
\text { interaction with small RNAs (siRNA, microRNAs, etc.) }\end{array}$ \\
\hline IncRNAWiki & Human & An open wiki style IncRNA database \\
\hline MONOCLdb & Mouse & $\begin{array}{l}\text { A mouse noncoding database detailing functional } \\
\text { enrichment of IncRNA in response to respiratory } \\
\text { disease caused by influenza and SARS-CoV }\end{array}$ \\
\hline IncRNome & Human & $\begin{array}{l}\text { A searchable database for long noncoding RNAs in humans } \\
\text { and various properties, such as predicted structure, SNPs } \\
\text { and epigenetic modifications }\end{array}$ \\
\hline PLncDB & Arabidopsis thaliana & $\begin{array}{l}\text { A database dedicated to A. thaliana plant IncRNA transcriptome, } \\
\text { including information on epigenetic modification }\end{array}$ \\
\hline $\begin{array}{l}\text { Functional IncRNA } \\
\text { Database }\end{array}$ & $\begin{array}{l}\text { Human, Mouse and } \\
\text { Rat }\end{array}$ & A database of experimentally validated functional IncRNAs \\
\hline $\ln C e D B$ & Human & A database of IncRNA acting as ceRNA \\
\hline Linc2GO & Human & $\begin{array}{l}\text { A database of IncRNA acting as ceRNA and biological processes } \\
\text { based on GO annotation }\end{array}$ \\
\hline IncRNASNP & Human and Mouse & $\begin{array}{l}\text { A database cataloging micro-RNA interactions and SNPs in } \\
\text { IncRNAs and their impact on secondary structure }\end{array}$ \\
\hline
\end{tabular}




\section{Genomic assays to study lincRNA regulations}

Methods to elucidate the functions of individual lincRNAs have made much slower progress compared to large-scale genomic assays. In this section we survey the increasing number of genome-scale molecular interaction studies to investigate the cellular functions of lincRNAs.

Several genomic approaches have been reported to identify specific functions of lincRNAs. One popular technique is the protein-centric RNA immunoprecipitation (RIP), which selects a particular protein or a group of proteins to co-precipitate RNAs and determines functional relationships based on physical interactions [41]. This allows one to ascribe functions of the protein(s) with co-precipitated lincRNAs. For example, Shi et al. used RIP to identify novel functional lincRNAs involved in the regulation of TNF expression through binding to PRC2 [42], and found that PRC2 binds to thousands of RNA species. Thus, protein-centric methods focusing on PRC2 have provided us critical insights into the genome-wide regulation by lincRNAs [43].

Conversely, another approach is to purify certain RNA molecules and then capture the associated proteins (RNA-centric methods); the associated proteins can then be identified via mass-spectroscopy [41]. This approach works by complementary base pairing of the RNA sequence to oligonucleotide probes labelled with streptavidin or biotin [44]. However, in comparison to protein-centric methods where the RNA targets can be amplified by PCR, RNA-centric methods do not have a means of amplifying the protein targets. Therefore, RNA-centric methods work best when large quantities of protein are available [41].

Additionally, there have also been a handful of "DNA-centric" methods for studying lincRNAs. Methods that investigate DNA modification or the 3D structure of chromosomes have greatly advanced our understanding of gene regulation [45]. For example, Ma et al. developed a novel method called Dnase Hi-C that determines the interactions of lincRNA promoters with DNA enhancer regions [45]. Their method involves cross-linking nearby DNA strands, followed by DNAse I digestion, proximity ligation between the cross-linked strands and DNA sequencing. Rather than using restrictive enzyme (RE) as done in conventional $\mathrm{Hi}-\mathrm{C}$, which generates predictable and consistent fragment ends, DNase I produces a heterogeneous mixture of fragment ends that greatly improves the efficiency and resolution. They were able to fine-map cell specific 3D organization of 998 lincRNA promoters. They demonstrated that lincRNA expression is tightly controlled by complex mechanisms including super-enhancers and PRCs.

\section{Known functions and mechanisms of lincRNAs}

Historically, lincRNAs have been shown to have a greater likelihood to be functionally associated with their nearest neighboring protein-coding genes. However, more recent analyses show that the expression correlation between a lincRNA and its closest coding gene is not statistically significant when compared to the correlation between two neighboring protein-coding genes $[8,46]$. While complementary base pairing may be the mechanism of action for some small RNAs such as microRNAs, lincRNAs by their nature are unlikely to exert their regulatory function solely through sequence pairing. Instead, lincRNAs have been shown to mediate the interplay between many molecular species simultaneously [47]. LincRNAs affect gene expression by many different mechanisms - 
from chromatin remodelling and epigenetic regulation, to transcriptional, posttranscriptional, and protein-level control. So far, no unifying genome-wide theme has been found to explain all the complexities of lincRNA regulation. We review the handful of competing theories that attempt to address this problem.

\section{LincRNAs involved in chromatin remodelling}

Epigenetics is a vital means of DNA patterning to regulate gene expression [48]. PRCs exert gene silencing epigenetically by histone modifications and DNA chemical alterations such as methylation [43]. Recruitment of PRCs to certain genomic locations is mediated by specific lincRNAs. Thus, the differential expression of certain lincRNAs (such as HOTAIR) can lead to activation or deactivation of transcription on the genome [49]. The vital role of gene suppression due to lincRNAs has been implicated in the pathology of cancers, where dysregulation of individual lincRNAs release cell cycle control resulting in an increase in cell proliferation [50]. Complicating matters, thousands of lincRNAs were found bound by PRC2 within various cell types [4], suggesting the widespread interaction of lincRNAs with the epigenetic modification machinery.

\section{LincRNAs as transcription co-factors}

Many lincRNAs are known to act as transcription co-factors. In some cases, the act of transcription of a lincRNA may positively or negatively affect expression of nearby genes [51]. Dimitrova et al. showed that lincRNA-p21 acts as a transcriptional coactivator and was required for recruitment of ribonucleoproteins to promoter elements associated with pre-mRNA [52]. MALAT1 is also known to act as a transcription co-factor. This lincRNA is well characterized as one of the most highly expressed mammalian lincRNAs. It is also known to significantly affect the metastasic process in lung adenocarcinoma, by enhancing the expression of cell motility genes [53]. It was found that MALAT1 acts as a molecular scaffold to allow gene expression by promoting the interaction among unmethylated PRC2, E2F1 transcription factor, histone markers, and the other transcriptional coactivator complexes [54]. Interestingly, this protein sequestration mechanism of ncRNA is not unique to eukaryotes, and it also occurs in bacteria [55].

\section{Competing endogenous RNA hypothesis of lincRNAs}

The competing endogenous RNA (ceRNA) hypothesis is a theory that lncRNAs (including lincRNAs) regulate gene expression by acting as microRNA sponges [56]. The inhibition of specific mRNA translation is modulated by microRNA depletion through lncRNAs harboring microRNA binding sites. By effectively competing for the same microRNA, these lncRNAs exert a level of competitive inhibition. Based on this hypothesis, Liu et al. developed a database of lincRNAs that were predicted to have functional associations with protein-coding genes [57]. Some exemplary lincRNAs that function as ceRNAs are the HULC [58] and LINC-ROR [59]. HULC was shown to be the molecular sponge of a series of microRNAs including miR-372, which induces phosphorylation of CREB in liver cancer [60], and LINC-ROR shares the microRNA response elements with core transcription factors Oct4, Sox2, and Nanog and thus increases expression of these genes by competing for microRNAs [61]. Although some lincRNAs act as ceRNAs, it is unclear how prevalent this mechanism is among all lincRNAs. 


\section{LincRNAs as evolutionary reservoirs}

While lincRNAs have less sequence conservation than protein-coding genes, they have a greater degree of secondary motif conservation compared to mRNAs [62]. These elements may explain the origins of lincRNAs, which provide a reservoir of evolutionarily constrained RNA motifs $[62,63]$ to supply extra genetic modules for evolutionary tinkering. It is also known that Retrotransposon and tandem repeat sequences are more common within lincRNAs compared to protein-coding genes [64]. Embedded microRNAs and the hypothesized ceRNA mechanism mentioned earlier may be accounted for by such duplication events, as modulating copy number of an embedded microRNA or target site would allow for fine-tuned regulation $[56,65]$.

\section{Computational methods for lincRNA target prediction}

There have been many attempts to computationally identify the function of lincRNAs. Given the length of lincRNA sequences and the complexity of their potential 3D structures along with the RNA and protein partners, this is a very challenging task. We review the different computational approaches in the following.

\section{Correlation with protein coding genes and biological processes}

One of the simplest approaches to determine the function of lincRNAs is to examine their correlations with protein coding genes [66]. However, this is a "black box" approach that identifies neither causality nor lincRNA functions at the molecular level. Another naïve approach is to relate the function of lincRNAs to the nearby protein coding genes [67]. Many lincRNAs have been found to exert regulatory activity on protein coding genes in cis $[45,52]$. However, Khalil et al. found that knockdown of six different lincRNAs did not affect the expression of level of nearby genes [4]. This suggests that lincRNAs can work in trans as well, and that the correlation between a lincRNA and its nearby protein coding genes may not necessarily be a causative relationship, but rather a result of sharing a region of active transcription.

\section{Relation between lincRNAs with microRNAs and other small non-coding RNAs}

Other more sophisticated tools have been developed to identify more succinct functions. Boerner and McGinnis constructed a pipeline to seek functions of lncRNAs in Zea Mays [33]. Using BLAST search, they found that the majority of lncRNAs have strong homology to small RNA molecules. They hypothesized that many lncRNAs are simply unprocessed pre-cursors to small non-coding RNAs, such as microRNA, shRNA and siRNA [33]. Based on the "ceRNA hypothesis" mentioned earlier, Liu et al. developed "linc2GO", a software for identifying mRNA and lincRNA pairs [57]. Using predicted microRNA targets from miRanda, TargetScan and PITA software, they predicted microRNA targets on both mRNAs and lincRNAs; The mRNAs and lincRNAs that had statistically significant target sites for a particular microRNA were proposed to have a "competing endogenous" relationship.

\section{Machine learning approaches to target and functional prediction}

Machine learning methods have been used successfully to classify whether transcripts are coding or non-coding. However, machine learning methods to identify the targets of lincRNAs have not seen much success. Comparatively, there has been much more 
success in using supervised learning approaches to identify microRNA targets, such as TargetScan [68], SvMicrO [69] and mirMark [70]. Still progress is being made towards lincRNA functional prediction. Glazko et al. used support vector machines (SVM) to predict lncRNA and PRC2 binding using human lincRNA associated with PRC2 as training data. With the classification model, they were able to predict $59.4 \%$ of $\operatorname{lncRNAs}$ which bind to PRC2 in mice [71]. The model was based off of the dataset by Khalil et al. $[4,72]$ which found roughly $20 \%$ of lincRNAs to associate with PRC2. However, it remains unclear whether the associations were spurious or led to sequence specific chromatin regulation.

\section{LincRNA functional prediction through the higher-order structure}

Perhaps the least explored lincRNA prediction approach is functional prediction through tertiary and quaternary structure. As the structure of RNA molecules are related to their functions, predicting the structure of complexes between RNA-RNA, and RNA-protein interactions could elucidate functional properties. Several RNA-RNA interaction prediction tools are available, usually based on free-energy, such as RNAhybrid [73] and RNADuplex [74]. RNA-protein interaction prediction tools exist as well, such as RPIseq which uses a Random Forest classification approach [75] or RNApred, which uses an SVM approach [76]. However, there have not been many attempts for lincRNA functional prediction. Many of the protein complexes interacting with lincRNAs do not fall into common binding motifs [41]. Furthermore, functional prediction is complicated by the "n-body problem", since protein, RNA and DNA can be complexed with lincRNAs simultaneously.

\section{Downstream target prediction through directed graphs}

Reverse engineering of gene regulatory networks has been an area of research before the explosion of next generation sequencing and lincRNA research [77]. Approaches such as Bayesian networks, information-theoretic approaches and ordinary differential equations have shown strong performance [78]. Generally, a perturbation of the system (such as gene knockout, overexpression or drug treatment) is performed which forces a node (i.e., a gene) on a regulatory network graph to be forcibly turned on or turned off. This perturbation produces direct causative (rather than correlative) downstream effects that can be captured through microarrays and quantitative methods. Recently, Jiang et al. published a database (lncRNA2Target) describing lncRNA knockdown and overexpression experiments, followed by gene quantification by microarray or qPCR [79]. These types of experiments can be a valuable resource for elucidating a lincRNA's targets and pathways.

\section{Conclusion}

Statistical evaluation studies for lincRNAs are urgently needed, as datasets produced by these various methods have thus far shown only modest overlaps in their identified lincRNAs [14]. Besides lack of sequence conservation among lincRNAs, another major issue hindering functional prediction is the lack of validated data. While there are many well-studied lincRNAs, there are massively more unannotated lincRNAs. Machine learning methods often require a large training dataset to produce accurate results. Several functional lincRNA/lncRNA databases exist (such as lncrnaDB), however the number of entries are very low and do not categorize the function of the lncRNAs in a systematic manner [80]. As more and more lincRNAs become functionally validated, comprehensive and 
regularly updated databases would be a great source to build good prediction methods. Perhaps even more important is the advancement of experimental techniques to provide quality data required for the prediction. Currently, most experimental techniques focus on a single protein or a small number of proteins (protein-centric) or a single lincRNA or family of lincRNAs (RNA-centric) [41]. New methods are required that can provide high-throughput protein and RNA targets of thousands of lincRNAs in parallel.

Competing interests

The authors declare that they have no competing interests.

\section{Authors' contributions}

LXG planned the work. TC, JM, JW and LXG all wrote parts of the manuscript. TC and LXG designed and finalized the manuscript. All authors read and approved the final manuscript.

\section{Acknowledgements}

This work was supported by grants K01ES025434 awarded by NIEHS through funds provided by the trans-NIH Big Data to Knowledge (BD2K) initiative (www.bd2k.nih.gov), P20 COBRE GM103457 awarded by NIH/NIGMS, and Medical Research Grant 14ADVC-64566 from Hawaii Community Foundation to L.X. Garmire.

\section{Author details}

${ }^{1}$ Molecular Biosciences and Bioengineering Graduate Program, University of Hawaii at Manoa, Honolulu, HI 96822, USA. ${ }^{2}$ Epidemiology Program, University of Hawaii Cancer Center, Honolulu, HI 96813, USA. ${ }^{3}$ Laboratory of Immunology and Signal Transduction, Chaminade University of Honolulu, Honolulu, HI 96816, USA. ${ }^{4}$ Department of Internal Medicine, University of lowa, lowa City, IA 52242, USA.

Received: 27 June 2015 Accepted: 4 December 2015

Published online: 22 December 2015

\section{References}

1. Hangauer MJ, Vaughn IW, McManus MT. Pervasive transcription of the human genome produces thousands of previously unidentified long intergenic noncoding RNAs. PLOS Genet. 2013;9(6):e1003569.

2. Ezkurdia I, Juan D, Rodriguez JM, Frankish A, Diekhans M, Harrow J, et al. Multiple evidence strands suggest that there may be as few as 19000 human protein-coding genes. Hum Mol Genet. 2014.

3. Joyce GF. The antiquity of RNA-based evolution. Nature. 2002;418(6894):214-21.

4. Khalil AM, Guttman M, Huarte M, Garber M, Raj A, Rivea Morales D, et al. Many human large intergenic noncoding RNAs associate with chromatin-modifying complexes and affect gene expression. Proc Natl Acad Sci U S A. 2009; 106(28):11667-72.

5. Volders P-J, Verheggen K, Menschaert G, Vandepoele K, Martens L, Vandesompele J, et al. An update on LNCipedia: a database for annotated human IncRNA sequences. Nucleic Acids Res. 2015;43(D1):D174-80.

6. Ulitsky I, Bartel DP. lincRNAs: genomics, evolution, and mechanisms. Cell. 2013;154(1):26-46.

7. Guttman M, Russell P, Ingolia NT, Weissman JS, Lander ES. Ribosome profiling provides evidence that large noncoding RNAs do not encode proteins. Cell. 2013;154(1):240-51.

8. Cabili MN, Trapnell C, Goff L, Koziol M, Tazon-Vega B, Regev A, et al. Integrative annotation of human large intergenic noncoding RNAs reveals global properties and specific subclasses. Genes Dev. 2011;25(18):1915-27.

9. Iyer MK, Niknafs YS, Malik R, Singhal U, Sahu A, Hosono Y, et al. The landscape of long noncoding RNAs in the human transcriptome. Nat Genet. 2015.

10. Mercer TR, Gerhardt DJ, Dinger ME, Crawford J, Trapnell C, Jeddeloh JA, et al. Targeted RNA sequencing reveals the deep complexity of the human transcriptome. Nat Biotechnol. 2012;30(1):99-104.

11. Sauvageau M, Goff LA, Lodato S, Bonev B, Groff AF, Gerhardinger C, et al. Multiple knockout mouse models reveal lincRNAs are required for life and brain development. Elife. 2013;2:e01749.

12. Ge X, Chen Y, Liao X, Liu D, Li F, Ruan H, et al. Overexpression of long noncoding RNA PCAT-1 is a novel biomarker of poor prognosis in patients with colorectal cancer. Med Oncol. 2013;30(2):1-6.

13. Guttman M, Garber M, Levin JZ, Donaghey J, Robinson J, Adiconis X, et al. Ab initio reconstruction of cell type-specific transcriptomes in mouse reveals the conserved multi-exonic structure of lincRNAs. Nat Biotechnol. 2010;28(5):503-U166.

14. Marques AC, Ponting CP. Catalogues of mammalian long noncoding RNAs: modest conservation and incompleteness. Genome Biol. 2009;10(11):R124.

15. Ning S, Wang P, Ye J, Li X, Li R, Zhao Z, et al. A global map for dissecting phenotypic variants in human lincRNAs. Eur J Hum Genet. 2013;21(10):1128-33.

16. Guttman M, Amit l, Garber M, French C, Lin MF, Feldser D, et al. Chromatin signature reveals over a thousand highly conserved large non-coding RNAs in mammals. Nature. 2009;458(7235):223-7.

17. Garmire LX, Garmire DG, Huang W, Yao J, Glass CK, Subramaniam S. A global clustering algorithm to identify long intergenic non-coding RNA-with applications in mouse macrophages. PLoS One. 2011;6(9):e24051.

18. Djebali S, Davis CA, Merkel A, Dobin A, Lassmann T, Mortazavi A, et al. Landscape of transcription in human cells. Nature. 2012;489(7414):101-8.

19. Yang L, Duff MO, Graveley BR, Carmichael GG, Chen L-L. Genomewide characterization of non-polyadenylated RNAs. Genome Biol. 2011;12(2):R16.

20. He Y, Vogelstein B, Velculescu VE, Papadopoulos N, Kinzler KW. The antisense transcriptomes of human cells. Science. 2008;322(5909):1855-7. 
21. Kawaji H, Lizio M, Itoh M, Kanamori-Katayama M, Kaiho A, Nishiyori-Sueki H, et al. Comparison of CAGE and RNA-seq transcriptome profiling using clonally amplified and single-molecule next-generation sequencing. Genome Res. 2014; 24(4):708-17.

22. Ulitsky I, Shkumatava A, Jan CH, Sive H, Bartel DP. Conserved function of lincRNAs in vertebrate embryonic development despite rapid sequence evolution. Cell. 2011;147(7):1537-50.

23. Tahira AC, Kubrusly MS, Faria MF, Dazzani B, Fonseca RS, Maracaja-Coutinho V, et al. Long noncoding intronic RNAs are differentially expressed in primary and metastatic pancreatic cancer. Mol Cancer. 2011;10:141.

24. Prensner JR, lyer MK, Balbin OA, Dhanasekaran SM, Cao Q, Brenner JC, et al. Transcriptome sequencing across a prostate cancer cohort identifies PCAT-1, an unannotated lincRNA implicated in disease progression. Nat Biotechnol. 2011;29(8):742-9.

25. Madden T. The BLAST sequence analysis tool. 2013.

26. Kong $L$, Zhang $Y, Y$ e ZQ, Liu XQ, Zhao SQ, Wei L, et al. CPC: assess the protein-coding potential of transcripts using sequence features and support vector machine. Nucleic Acids Res. 2007;35(Web Server issue):W345-349.

27. Sun $K$, Chen $X$, Jiang $P$, Song $X$, Wang H, Sun $H$. iSeeRNA: identification of long intergenic non-coding RNA transcripts from transcriptome sequencing data. BMC Genomics. 2013;14 Suppl 2:S7.

28. Jalali $\mathrm{S}$, Jayaraj GG, Scaria $\mathrm{V}$. Integrative transcriptome analysis suggest processing of a subset of long non-coding RNAs to small RNAs. Biol Direct. 2012;7:25.

29. Sakharkar MK, Chow VT, Kangueane P. Distributions of exons and introns in the human genome. In Silico Biol. 2004;4(4):387-93.

30. Semon M, Duret L. Evidence that functional transcription units cover at least half of the human genome. Trends Genet. 2004;20(5):229-32.

31. Qiu MT, Hu JW, Yin R, Xu L. Long noncoding RNA: an emerging paradigm of cancer research. Tumour Biol. 2013; 34(2):613-20.

32. Wang Y, Li Y, Wang Q, LV Y, Wang S, Chen X, et al. Computational identification of human long intergenic non-coding RNAs using a GA-SVM algorithm. Gene. 2014;533(1):94-9.

33. Boerner S, McGinnis KM. Computational identification and functional predictions of long noncoding RNA in Zea mays. PLoS One. 2012;7(8):e43047.

34. Fan X-N, Zhang S-W. IncRNA-MFDL: identification of human long non-coding RNAs by fusing multiple features and using deep learning. Mol Biosyst. 2015. 11.3 (2015):892-897.

35. Xie C, Yuan J, Li H, Li M, Zhao G, Bu D, et al. NONCODEv4: exploring the world of long non-coding RNA genes. Nucleic Acids Res. 2014;42(D1):D98-D103.

36. Flicek P, Amode MR, Barrell D, Beal K, Brent S, Carvalho-Silva D, et al. Ensembl 2012. Nucleic Acids Res. 2012; 40(Database issue):D84-90.

37. Harrow J, Frankish A, Gonzalez JM, Tapanari E, Diekhans M, Kokocinski F, et al. GENCODE: the reference human genome annotation for The ENCODE Project. Genome Res. 2012;22(9):1760-74.

38. Hsu F, Kent WJ, Clawson H, Kuhn RM, Diekhans M, Haussler D. The UCSC known genes. Bioinformatics. 2006;22(9): 1036-46.

39. Griffiths-Jones S, Moxon S, Marshall M, Khanna A, Eddy SR, Bateman A. Rfam: annotating non-coding RNAs in complete genomes. Nucleic Acids Res. 2005;33 suppl 1:D121-4.

40. Dunham I, Kundaje A, Aldred SF, Collins PJ, Davis CA, Doyle F, et al. An integrated encyclopedia of DNA elements in the human genome. Nature. 2012;489(7414):57-74.

41. McHugh CA, Russell P, Guttman M. Methods for comprehensive experimental identification of RNA-protein interactions. Genome Biol. 2014;15:203.

42. Shi L, Song L, Fitzgerald M, Maurer K, Bagashev A, Sullivan KE. Noncoding RNAs and LRRFIP1 regulate TNF expression. J Immunol. 2014;192(7):3057-67.

43. Goff LA, Rinn JL. Poly-combing the genome for RNA. Nat Struct Mol Biol. 2013;20(12):1344-6.

44. Gong C, Maquat LE. Affinity Purification of Long Noncoding RNA-Protein Complexes from Formaldehyde Cross-Linked Mammalian Cells. In: Regulatory Non-Coding RNAs. edn.: Springer; New York. 2015: 81-86.

45. Ma W, Ay F, Lee C, Gulsoy G, Deng X, Cook S, et al. Fine-scale chromatin interaction maps reveal the cisregulatory landscape of human lincRNA genes. Nat Methods. 2014.

46. Wright MW. A short guide to long non-coding RNA gene nomenclature. Hum Genomics. 2014;8:7.

47. Mercer TR, Dinger ME, Mattick JS. Long non-coding RNAs: insights into functions. Nat Rev Genet. 2009;10(3):155-9.

48. Di Croce L, Helin K. Transcriptional regulation by Polycomb group proteins. Nat Struct Mol Biol. 2013;20(10):1147-55.

49. Loewen G, Zhuo Y, Zhuang Y, Jayawickramarajah J, Shan B. lincRNA HOTAIR as a novel promoter of cancer progression. J Can Res Updates. 2014;3(3):134-40.

50. Gutschner T, Diederichs S. The hallmarks of cancer: a long non-coding RNA point of view. RNA Biol. 2012;9(6):703-19.

51. Wilusz JE, Sunwoo H, Spector DL. Long noncoding RNAs: functional surprises from the RNA world. Genes Dev. 2009;23(13):1494-504.

52. Dimitrova N, Zamudio JR, Jong RM, Soukup D, Resnick R, Sarma K, et al. LincRNA-p21 activates p21 in cis to promote Polycomb target gene expression and to enforce the G1/S checkpoint. Mol Cell. 2014;54(5):777-90.

53. Wang KC, Chang HY. Molecular Mechanisms of Long Noncoding RNAs. Mol Cell. 2011;43(6):904-14.

54. Yang L, Lin C, Liu W, Zhang J, Ohgi KA, Grinstein JD, et al. ncRNA- and PC2 methylation-dependent gene relocation between nuclear structures mediates gene activation programs. Cell. 2011;147(4):773-88.

55. Duss O, Michel E, Yulikov M, Schubert M, Jeschke G, Allain FHT. Structural basis of the non-coding RNA RsmZ acting as a protein sponge. Nature. 2014;509(7502):588-+.

56. Salmena L, Poliseno L, Tay Y, Kats L, Pandolfi PP. A ceRNA hypothesis: the Rosetta Stone of a hidden RNA language? Cell. 2011;146(3):353-8.

57. Liu K, Yan Z, Li Y, Sun Z. Linc2GO: a human LincRNA function annotation resource based on ceRNA hypothesis. Bioinformatics. 2013;29(17):2221-2. 
58. Xie H, Ma H, Zhou D. Plasma HULC as a promising novel biomarker for the detection of hepatocellular carcinoma. BioMed research international 2013. 2013.

59. Zhou X, Gao Q, Wang J, Zhang X, Liu K, Duan Z. Linc-RNA-RoR acts as a "sponge" against mediation of the differentiation of endometrial cancer stem cells by microRNA-145. Gynecologic oncology. 2014; 133(2):333-339.

60. Wang J, Liu X, WU H, Ni P, Gu Z, Qiao Y, et al. CREB up-regulates long non-coding RNA, HULC expression through interaction with microRNA-372 in liver cancer. Nucleic Acids Res. 2010:38(16):5366-83.

61. Wang $Y, X u Z$, Jiang J, Xu C, Kang J, Xiao L, et al. Endogenous miRNA sponge lincRNA-RoR regulates Oct4, Nanog, and Sox2 in human embryonic stem cell self-renewal. Dev Cell. 2013;25(1):69-80.

62. Kapusta A, Feschotte C. Volatile evolution of long noncoding RNA repertoires: mechanisms and biological implications. Trends Genet. 2014;30(10):439-52.

63. Smith MA, Gesell T, Stadler PF, Mattick JS. Widespread purifying selection on RNA structure in mammals. Nucleic Acids Res. 2013;41(17):8220-36.

64. Kelley D, Rinn J. Transposable elements reveal a stem cell-specific class of long noncoding RNAs. Genome Biol. 2012;13(11):R107.

65. Labialle S, Cavaillé J. Do repeated arrays of regulatory small-RNA genes elicit genomic imprinting? Bioessays. 2011; 33(8):565-73.

66. Guo X, Gao L, Liao Q, Xiao H, Ma X, Yang X, et al. Long non-coding RNAs function annotation: a global prediction method based on bi-colored networks. Nucleic Acids Res. 2013;41(2):e35.

67. Ma H, Hao Y, Dong X, Gong Q, Chen J, Zhang J, et al. Molecular mechanisms and function prediction of long noncoding RNA. Sci World J. 2012;2012.

68. Lewis BP, Burge CB, Bartel DP. Conserved seed pairing, often flanked by adenosines, indicates that thousands of human genes are microRNA targets. Cell. 2005;120(1):15-20.

69. Liu H, Yue D, Chen Y, Gao S-J, Huang Y. Improving performance of mammalian microRNA target prediction. BMC Bioinformatics. 2010;11(1):476.

70. Menor M, Ching T, Zhu X, Garmire D, Garmire LX. mirMark: a site-level and UTR-level classifier for miRNA target prediction. Genome Biol. 2014;15(10):500

71. Glazko GV, Zybailov BL, Rogozin IB. Computational prediction of polycomb-associated long non-coding RNAs. PLoS One. 2012;7(9):e44878.

72. Felekkis K, Voskarides K. Genomic Elements in Health, Disease and Evolution. 2015

73. Krüger J, Rehmsmeier M. RNAhybrid: microRNA target prediction easy, fast and flexible. Nucleic Acids Res. 2006;34 suppl 2:W451-4.

74. Hofacker, Ivo L. "Fast folding and comparison of RNA secondary structures." Monatshefte für Chemie/Chemical Monthly 125.2 (1994): 167-188.

75. Muppirala U, Lewis BA, Dobbs D. Computational tools for investigating RNA-protein interaction partners. J Comput Sci Syst Biol. 2013;6:182-7.

76. Kumar M, Gromiha MM, Raghava GP. SVM based prediction of RNA-binding proteins using binding residues and evolutionary information. J Mol Recognit. 2011;24(2):303-13.

77. Murphy K, Mian S. Modelling gene expression data using dynamic Bayesian networks, In.: Technical report, Computer Science Division. Berkeley: University of California; 1999.

78. Bansal M, Belcastro V, Ambesi-Impiombato A, Di Bernardo D. How to infer gene networks from expression profiles. Mol Syst Biol. 2007;3(1):78.

79. Jiang Q, Wang J, Wu X, Ma R, Zhang T, Jin S, et al. LncRNA2Target: a database for differentially expressed genes after IncRNA knockdown or overexpression. Nucleic Acids Res. 2015;43(D1):D193-6.

80. Galperin MY, Rigden DJ, Fernández-Suárez XM. The 2015 Nucleic Acids Research Database Issue and Molecular Biology Database Collection. Nucleic Acids Res. 2015;43(D1):D1-5.

\section{Submit your next manuscript to BioMed Central and we will help you at every step:}

- We accept pre-submission inquiries

- Our selector tool helps you to find the most relevant journal

- We provide round the clock customer support

- Convenient online submission

- Thorough peer review

- Inclusion in PubMed and all major indexing services

- Maximum visibility for your research

Submit your manuscript at www.biomedcentral.com/submit 